\title{
Optimal management after paediatric lumbar puncture: a randomized controlled trial
}

\author{
Bing $\mathrm{Hu}^{1 \dagger}$, Tian-ming Chen ${ }^{1 \dagger}$, Bing Liu ${ }^{1}$, Wei Chi ${ }^{1}$, Yi-qing Miao ${ }^{1}$, Xiao-lu Nie², Xiao-xia Peng ${ }^{2^{*+}}$ and Gang Liu ${ }^{1{ }^{*+}}$
}

\begin{abstract}
Background: To evaluate whether a shorter time of lying supine without a pillow and fasting for solids and liquids (LSFSL) after a lumbar puncture (LP) is associated with a higher risk of post-lumbar puncture headache (PLPH) and post-lumbar puncture lower back pain (PLPBP) in a randomized, assessor-blinded, controlled trial.

Methods: Paediatric patients who underwent their first LP after hospital admission were randomly allocated to either the group with half an hour of LSFSL (0.5 h LSFSL) or $4 \mathrm{~h}$ of LSFSL (4 h LSFSL) immediately after LP. The primary outcome is PLPH after LP. The incidence of PLPH, PLPBP, and vomiting; vital signs (respiratory rate, heart rate, blood pressure); and other post-procedure conditions after LP were measured as the outcomes. The Noninferiority test and Wilcoxon rank-sum test were used to analyse the outcome data.
\end{abstract}

Results: In total, 400 patients (201 in the 0.5-h LSFSL group and 199 in the 4-h LSFSL group) were included in this trial. Twelve (5.97\%) of 201 patients experienced PLPH in the $0.5 \mathrm{~h}$ LSFSL group versus 13 (6.53\%) of 199 patients in the $4 \mathrm{~h}$ LSFSL group (difference $0.56,95 \% \mathrm{Cl}-4.18$ to $5.31 ; p=0.0108$ for the non-inferiority test). Fourteen (6.97\%) of 201 patients experienced PLPBP in the $0.5 \mathrm{~h}$ LSFSL group versus 17 (8.54\%) of 199 patients in the $4 \mathrm{~h}$ LSFSL group (difference $1.57,95 \% \mathrm{Cl}-3.66$ to $6.82 ; p=0.007$ for the non-inferiority test). The changes in heart rate (HR), respiratory rate (RP) and systolic blood pressure (SBP) before and after the LP were not different between the 0.5-h LSFSL group and the 4-h LSFSL group. No other adverse events were reported.

Conclusions: Compared with $4 \mathrm{~h}$ of LSFSL after LP, $0.5 \mathrm{~h}$ of LSFSL was not associated with a higher risk of PLPH, PLPBP or other adverse events. In conclusion, $0.5 \mathrm{~h}$ of LSFSL is sufficient for children undergoing LP.

Trial registration: Clinical trial NCT02590718. The date of registration was 08/25/2015.

Keywords: Lumbar puncture, Duration of bed rest, Supine position, Postoperative complications

\section{Background}

Lumbar puncture (LP) is a common clinical procedure that is widely used in the diagnosis of central nervous system infections, intracranial autoimmune diseases, subarachnoid haemorrhage, and intracranial tumours. Post-lumbar puncture headache (PLPH) and post-lumbar

\footnotetext{
*Correspondence: pengxiaoxia@bch.com.cn; niuniu@ccmu.edu.cn; liugangbch@sina.com

'Bing Hu and Tian-ming Chen contributed equally to this work and should be regarded as co-first authors.

${ }^{2}$ Center for Clinical Epidemiology and Evidence-based Medicine, Beijing Children's Hospital, Capital Medical University, Nalishi Road 56\#, Xicheng District, Beijing 100045, China

'Department of Infectious Diseases, Beijing Children's Hospital, Capital Medical University, Nalishi Road 56\#, Xicheng District, Beijing 100045, China
}

puncture lower back pain (PLPBP) are the most common post-procedure complications [1]. PLPH is a condition thought to result from failure of the dural puncture site to close, leading to cerebrospinal fluid (CSF) leakage and intracranial hypovolemia. This causes traction on the pain-sensitive structures in the brain, resulting in a headache $[2,3]$. Other factors correlated with the occurrence of PLPH include age, sex, body mass index, the needle gauge, the shape of the needle point, the needle orientation, the direction of the needle bevel, withdrawal of the needle core, and the operator skill level $[4,5]$. In domestic and foreign studies, no significant correlation was found between the duration of bed rest in the supine position without a pillow immediately after the LP and the

(c) The Author(s). 2019 Open Access This article is distributed under the terms of the Creative Commons Attribution 4.0 International License (http://creativecommons.org/licenses/by/4.0/), which permits unrestricted use, distribution, and 
occurrence of post- procedure complications, including PLPH. Due to the lack of a standard duration of bed rest after LP in China, most patients are advised to lie down for 4-6 h. It can be challenging to obtain cooperation with bed rest from paediatric patients for that length of time. In the meanwhile, fasting for solids and liquids may cause low blood sugar, leading to complications and inconvenience for both patients and doctors. In this randomized controlled clinical trial, we studied whether the duration of bed rest in the supine position without a pillow while fasting for solids and liquids (LSFSL) is associated with PLPH and PLPBP.

\section{Methods}

\section{Setting}

This research was conducted at the Infectious Disease Department of Beijing Children's Hospital between March 2015 and December 2017. It was approved (No. 2015-2) by the Ethics Committee of Beijing Children's Hospital affiliated with Capital Medical University. The protocol (ID: 20150826) was registered in ClinicalTrials.gov.

\section{Participants}

We recruited participants undergoing their first LP after admission. The inclusion criteria were as follows: 29 days to 18 years old, normal mental status, and no contraindications for LP. The exclusion criteria were as follows: contraindication for LP, mental retardation or mental symptoms, incapable of mobilization after LP, signs of unconsciousness, lidocaine allergy, history of headache and lower back pain before LP, prior LP.

\section{Randomization and blinding}

An independent statistician prepared randomization numbers using a computer-generated random assignment procedure. The results of the randomization allocation were kept in envelopes. Each envelope was designated for one participant. The envelopes were opened when he participants joined the trial. All participants were informed that there was a $50 \%$ chance of being assigned to the 0.5 -h LSFSL group or the 4-h LSFSL group. The data were analysed by an independent statistician who was blinded to the participants' allocation.

\section{Interventions}

All LPs were performed by two experienced clinicians. To avoid possible bias caused by practitioner interaction, equal numbers of participants who were assigned to the 0.5 -h and 4-h LSFSL groups were randomly allocated to each clinician. The LP procedures strictly followed the instructions in Zhu Fu-tang Practical Paediatrics (8th ed.) [6]. A standard LP package with the 22 gauge puncture needle was used for patients all the patients. All the patients had no pre-procedure sedation. All the patients were placed in a lateral knee-chest position, and the needle was inserted parallel to the floor with the bevel pointing upwards; $3-10 \mathrm{ml}$ of CSF was collected, the needle core was inserted, and the needle was withdrawn. Patients then underwent $0.5 \mathrm{~h}$ or $4 \mathrm{~h}$ of LSFSL after the LP, according to their assigned group.

\section{Outcomes}

A study nurse in the ward observed the patients for symptoms of headache, lower back pain, nausea and cerebral hernia during the 5 days after the LP. When symptoms occurred, the time and treatment were recorded. Our nurse assessed the patients from $4 \mathrm{~h}$ to 5 days after LP every $8 \mathrm{~h}$ every day. If the patients have discomfort complaint at any time, we will also make an assessment immediately. Patients less than 2 years old can't describe their pain. The Face, Legs, Activity, Cry, and Consolability (FLACC) behavioral pain scale was previously found to have excellent validity and reliability for pain assessment in young, cognitively intact children [7]. So we used FLACC to access the patients less than 2 years old whether they had pain or not. Our study nurse observed these patients for at least 1-2 min from $4 \mathrm{~h}$ to 5 days after LP every $8 \mathrm{~h}$ every day. Vitals were monitored hourly for $4 \mathrm{~h}$ after the LP. The results are presented in the CRF table. All the doctors and the study nurse were specially trained before participating in this study. For the diagnosis of PLPH, we followed the guidelines of The International Headache Society and the International Classification of Headache (ICHD-II). PLPH was defined as a headache occurring within 5 days of the LP and disappearing spontaneously within a week or within $48 \mathrm{~h}$ of the successful treatment of CSF leakage; PLPHs intensify after 15 min of sitting or standing up from a recumbent position and remits within $15 \mathrm{~min}$ of lying down. The definition of PLPBP was persistent pain surrounding the location of the puncture $[4,8]$. Patients' heart rates (HR), respiratory rates (RR), and systolic blood pressure (SBP) before and after the LP were also recorded.

\section{Withdrawal from the study}

Participants were free to withdraw at any time without having to provide a reason during the entire trial period.

\section{Adverse events}

Participants were instructed to record any unexpected signs or symptoms in CRFs during the trial period. All participating doctors and study nurses were required to record any observed adverse events (AEs).

\section{Statistical methods}

The primary outcomes were analysed by non-inferiority test (Confidence interval method) and The changes in 
HR, RP and SBP before and after the LP between two groups were analysed by Student's $t$ test. The baseline binary variable of sex was compared by the $x^{2}$ test and other baseline continuous variables were compared by Wilcoxon test if the normal distribution were not satisfied. If $p$ value was $<0.05$, the differences were statistically significant except for non-inferiority test, which the significance level of the test was 0.025. All statistical analyses were conducted using SPSS 17.0 (SPSS Inc., Chicago, IL, USA) and JMP 14.0.

\section{Sample size calculation}

PASS 16.0 was used to calculate the sample sizes. Sample sizes of 171 in $0.5 \mathrm{~h}$ LSFSL group and 171 in $4 \mathrm{~h}$ LSFSL achieved $80.07 \%$ power to detect a non-inferiority margin difference between the group proportions of 0.05.The reference group ( $4 \mathrm{~h}$ LSFSL) proportion of headache was $15 \%$ while the treatment group $(0.5 \mathrm{~h}$ LSFSL) proportion was $20 \%$ under the null hypothesis of inferiority. The power was computed for the case when the actual treatment group proportion was $10 \%$. The test statistic used is the one-sided $\mathrm{Z}$ test. The significance level of the test was 0.025 . We intended to recruit 200 participants for each group to allow for a rate of loss to follow-up of $10 \%$.

\section{Results}

Participants and baseline characteristics

In total, 400 inpatients were enrolled. They were randomized to the 0.5-h LSFSL group $(n=201)$ and the 4-h LSFSL group $(n=199)$. A flow diagram of the trial procedure is shown in Fig. 1. CSF was successfully collected from all 400 patients, which is an LP success rate of $100 \%$; $92 \%$ of patients (368 patients) were one attempt. Other $8 \%$ (32 patients) were 2-4 attempts.

There were no significant differences between the 2 groups with regard to the participants' age, sex, or amount of CSF collected. All patient demographics were comparable between the two groups. The results are shown in Table 1 . The vital signs (respiratory rate, pulse, heart rate, blood pressure) of all patients were within the normal ranges before they underwent the LP.

\section{Outcomes and estimation}

The FLACC scores of the patients who were under 2 years old show no pain from $4 \mathrm{~h}$ to 5 days after LP. So all the patients younger than 2 years didn't suffer PLPH and PLPBP. The rate of PLPH was 5.97\% (95\% CI 3.12 to 10.2 ) in $0.5 \mathrm{~h} \mathrm{LSFSL}$ group and $6.53 \%$ (95\% CI 3.52 to 10.9) in $4 \mathrm{~h}$ LSFSL group. The one-sided $95 \% \mathrm{CI}$ for the underpowered non-inferiority test on the rate difference

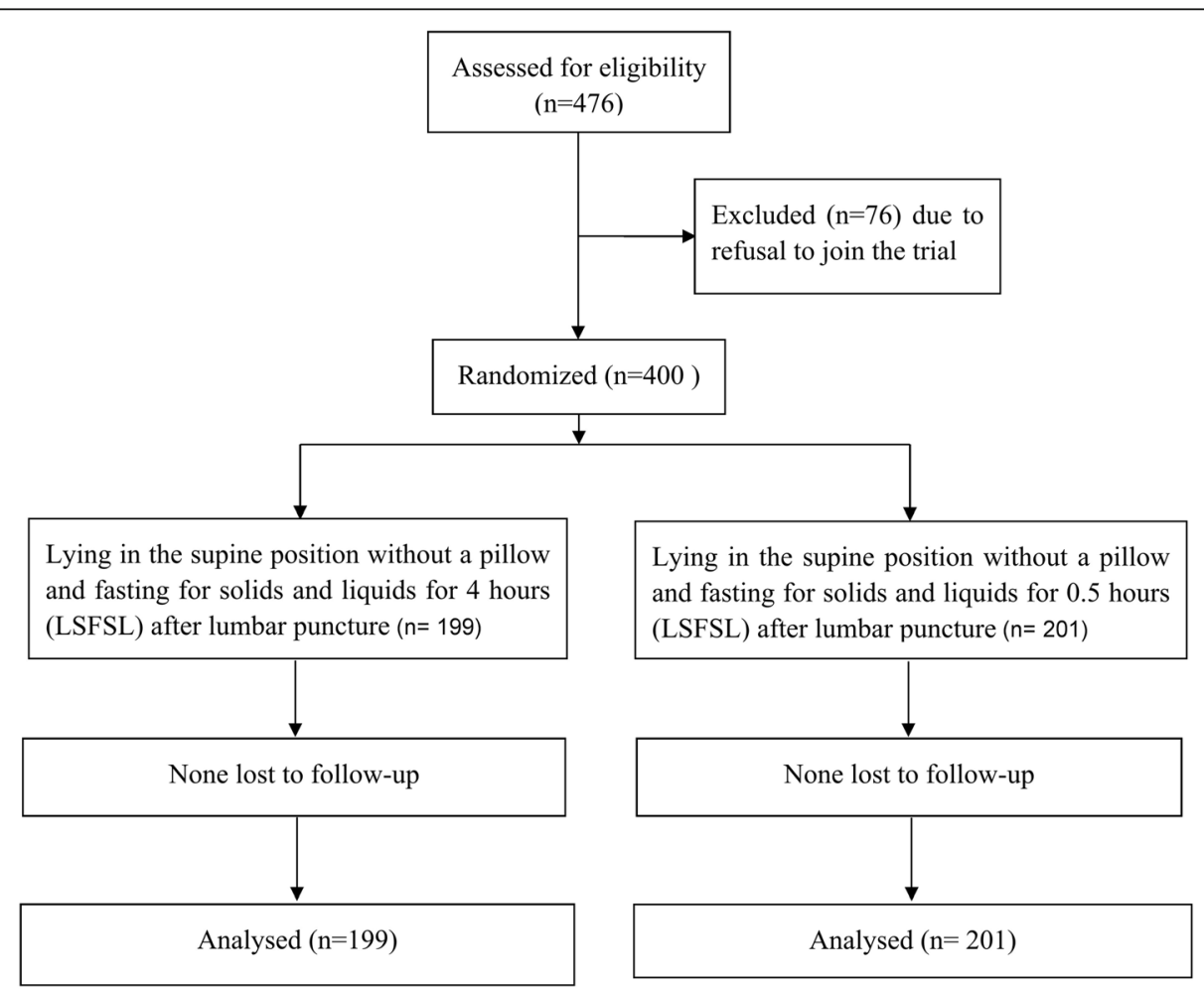

Fig. 1 Flow diagram of the trial procedure. 0.5-h LSFSL: lying in the supine position without a pillow and fasting for solids and liquids for half an hour after lumbar puncture; 4-h LSFSL: lying in the supine position without a pillow and fasting for solids and liquids for $4 \mathrm{~h}$ after lumbar puncture; HR: heart rate; RR: respiratory rate; SBP: systolic blood pressure 
Table 1 Demographic characteristics of the included participants

\begin{tabular}{|c|c|c|c|c|}
\hline & 0.5 h LSFSL $(n=201)$ & $\begin{array}{l}4 \mathrm{~h} \text { LSFSL } \\
(n=199)\end{array}$ & test value & $P$ \\
\hline Sex & & & $3.242^{*}$ & 0.073 \\
\hline Male, n(\%) & $120(59.7)$ & 136(68.3) & & \\
\hline Female, $n(\%)$ & $81(40.3)$ & 63(31.7) & & \\
\hline Age(y), median [IQR] & $1.0(0.3-6.0)$ & $2.0(0.3-6.0)$ & $0.191^{\#}$ & 0.658 \\
\hline \multicolumn{5}{|l|}{ Vital signs before LP } \\
\hline RR(per minute), median [IQR] & $26(22-31)$ & $26(22-31)$ & $0.269^{\#}$ & 0.604 \\
\hline HR(per minute), median [IQR] & 120(98-132) & 120(100-135) & $0.293^{\#}$ & 0.588 \\
\hline $\mathrm{SBP}(\mathrm{mmHg})$, median [IQR] & $90(80-96.5)$ & $90(80-96)$ & $0.057^{\#}$ & 0.810 \\
\hline Number of attempts, median [IQR] & $1(1-1)$ & $1(1-1)$ & $0.002^{\#}$ & 0.963 \\
\hline Amount of CSF(ml), median [IQR] & $5(3-5)$ & $5(3-5)$ & $1.021^{\#}$ & 0.312 \\
\hline
\end{tabular}

LSFSL lying in the supine position without a pillow and fasting for solids and liquids, $H R$ heart rate, $R R$ respiratory rate, SBP systolic blood pressure, CSF cerebral spine fluid

*t value

\#z value

was $0.56 \%$ ( $95 \%$ CI -4.18 to 5.31 ), with a non-inferiority margin of $5 \%$. The results are shown in Table 2.

The rate of PLPBP was $6.97 \%$ (95\% CI 3.86 to 11.4) in $0.5 \mathrm{~h}$ LSFSL group and $8.54 \%$ (95\% CI 5.06 to 13.3 ) in 4 $\mathrm{h}$ LSFSL group. The one-sided 95\% CI for the underpowered non-inferiority test on the rate difference was $1.57 \%$ (95\% CI -3.66 to 6.82 ), with a non-inferiority margin of $5 \%$. The results are shown in Table 2 .

All patients' vital signs (respiratory rate, pulse, heart rate, blood pressure) were within the normal ranges $4 \mathrm{~h}$ after they underwent the LP. The changes in HR, RP and SBP before and after the LP were $-0.144 \pm 10.641$ (per minute), $-0.408 \pm 3.493$ (per minute), $0.503 \pm 6.241$ $(\mathrm{mmHg})$ respectively in $0.5 \mathrm{~h}$ LSFSL group and $-1.070 \pm$ 10.214 (per minute), $-0.347 \pm 3.755$ (per minute), 1.397 $\pm 6.692(\mathrm{mmHg})$ respectively in $4 \mathrm{~h}$ LSFSL group. The changes in HR, RP and SBP before and after the LP were not significantly different between the $0.5-\mathrm{h}$ and 4-h LSFSL groups. The results are shown in Fig. 2. No other AEs were reported during the trial.

\section{Discussion}

Our research suggests that in paediatric patients, LSFSL for $30 \mathrm{~min}$ or $4 \mathrm{~h}$ result in equivalent incidence rates of PLPH and PLPBP, and the duration of LSFSL does not affect the patient's vital signs. However, prolonged LSFSL can be inconvenient for both the patients and the medical staff. Standardized instructions should be strictly followed to improve the LP procedure in children.

LP is a basic clinical procedure that is widely used in paediatric medicine. The procedure is safe when the instructions are strictly followed. However, complications can occur, and PLPH is the most common complication [1]. The incidence of PLPH varies in domestic and foreign research papers. Ren Bo analysed 198 children who underwent LP and reported a PLPH incidence rate of $5.05 \%$ and a PLPBP incidence rate of $11.62 \%$ [9]. This result is similar to ours. In foreign research papers, the incidence of PLPH was found to be approximately $40 \%$ (1-70\%) [10], and according to a retrospective analysis of foreign research papers by Yen-Feng Wang [3], the incidence of PLPH reached approximately $1 / 3$ of patients undergoing LP.

It is believed that PLPH is caused by intracranial hypotension due to the leakage of CSF after LP $[3,4]$. According to the review by Yan Wei Ling, PLPH has no significant correlation with either age or sex. A smaller amount of collected CSF $(<15 \mathrm{ml})$ and the number of previous LPs had also no significant correlations with PLPH [11]. Other factors correlated with PLPH include the following: needle gauge, the shape of the needle point, the needle orientation, the direction of the needle

Table 2 Incidence of headache or back pain after lumber puncture

\begin{tabular}{llllllll}
\hline Events & $\begin{array}{l}0.5 \mathrm{~h} \mathrm{LSFSL} \\
(n=201)\end{array}$ & $\begin{array}{l}4 \mathrm{~h} \mathrm{LSFSL} \\
(n=199)\end{array}$ & $Z$ value & $P$ value & Non-inferiority margin & Rate difference & $95 \%$ confidence interval \\
\hline PLPH, n(\%) & $12(5.97 \%)$ & $13(6.53 \%)$ & 2.298 & 0.011 & -0.05 & 0.0056 & -0.0418 to 0.0531 \\
PLPBP, n (\%) & $14(6.97 \%)$ & $17(8.54 \%)$ & 2.460 & 0.007 & -0.05 & 0.0157 & -0.0366 to 0.0682 \\
Composite endpoint, $\mathrm{n}(\%)$ & $24(11.94 \%)$ & $25(12.56 \%)$ & 1.715 & 0.043 & -0.05 & 0.0062 & -0.058 to 0.0705 \\
\hline
\end{tabular}

LSFSL lying in the supine position without pillow and fasting for solids and liquids, PLPBP post lumbar puncture lower back pain, $P L P H$ post lumbar puncture headache 


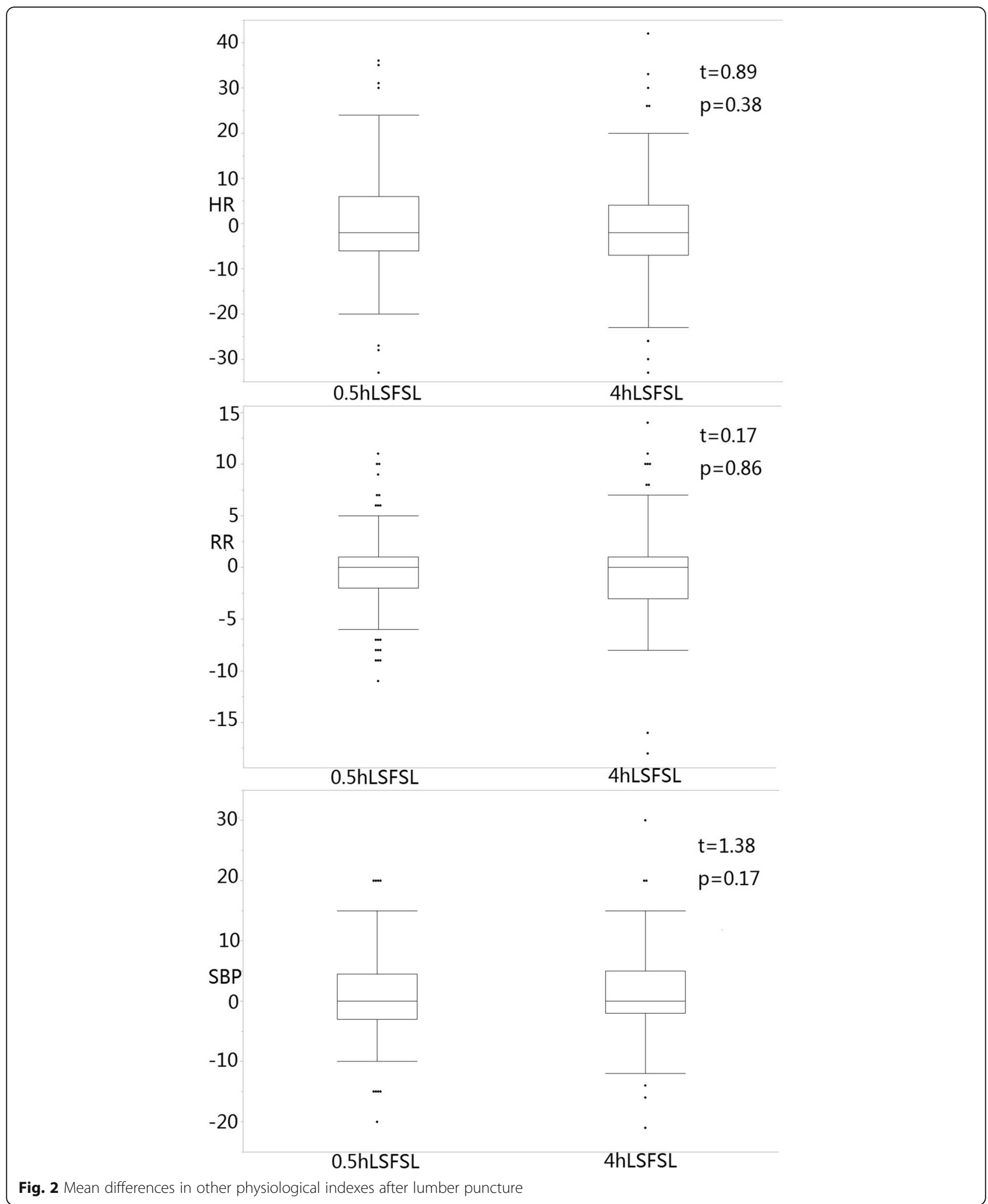

bevel, withdrawal of the needle core, and operator skill level $[4,5]$. It has been observed that the model of needle used significantly affects the incidence of PLPH. The larger the needle, the higher the incidence rate of PLPH is $[8,12,13]$. In China, the No.7 puncture needle $(0.7$ $\mathrm{mm}$ in diameter) with a lead bevel is the common choice 
for LP. For neonates and infants, the No. 5.5 puncture needle $(0.55 \mathrm{~mm}$ in diameter) has been recommended by previous studies, as it increases the success rate of LP and reduces haemorrhage, with the only disadvantage being the loss of the ability to measure the cerebrospinal fluid pressure (CSFP) [14]. In our study, we standardized the factors influencing PLPH by using a standard LP package with 22 gauge puncture needle, arranging for clinicians of the same skill level to perform the LPs, using the same puncture angle with the bevel facing upwards and always withdrawing the needle with the core inside. Also the amount of CSF collected from each participant ranged from 3 to $10 \mathrm{ml}$. The results showed a PLPH incidence rate of $6.25 \%$ and a PLPBP incidence rate of $7.75 \%$; no instances of cerebral hernia or other severe complications occurred during the research. These results are in agreement with the statistics reported in domestic and foreign research papers.

At present, the treatment for PLPH is LSFSL immediately after the LP, but there is no standard management guide for post-LP patients. In clinical practice, patients are routinely advised to LSFSL for $4-6 \mathrm{~h}$ after the LP to prevent PLPH and PLPBP [15]. According to Zhu Fu-tang Practical Paediatrics (8th ed.), the patient should lie supine position without a pillow for 30-60 min after a LP, and fasting is not mentioned. After a reviewing domestic and foreign studies, we found no evidence that supports the idea that a longer duration of bed rest reduces the risk of PLPH [16-20]. In contrast, a study has showed that a longer duration of bed rest could be related to a higher incidence of PLPH. In China, Jin Xiao Ping's research on adult LPs and Yan Wei Ling's study on paediatric LPs found no significant correlation between the duration of post-LP bed rest and the incidence of PLPH $[11,21]$. In agreement with the studies above, our study revealed that $0.5 \mathrm{~h}$ of LSFSL was not associated with a higher risk of PLPH, PLPBP or other adverse events, compared with $4 \mathrm{~h}$ of LSFSL after LP. This finding demonstrated that the duration of LSFSL was not correlated with the incidence of PLPH. Since it is difficult for paediatric patients, especially for infants, to cooperate with remaining still in the supine position without a pillow for hours, long periods of post-LP bed rest has often been an inconvenience for both the patients and the medical staff. The results of this research indicate that in the future, it is only necessary to maintain 30 min of LSFSL after the LP, instead of $4-6 \mathrm{~h}$.

In this research, the enrolled patients younger than 2 years old can't clearly describe headache or lower back ache. This might be a limitation, but FLACC score can be used to assess pain from burns and other etiologies for preverbal children and was previously found to have excellent validity and reliability for pain assessment in young, cognitively intact children. So our result is reliable to a great extent.

\section{Conclusions}

In conclusion, compared with $4 \mathrm{~h}$ of LSFSL after LP, 0.5 $h$ of LSFSL is not associated with higher incidence rates of PLPH, PLPBP or other adverse event. In conclusion, $0.5 \mathrm{~h}$ of LSFSL is adequate for children who have undergone a LP.

\begin{abstract}
Abbreviations
0.5-h LSFSL: Lying in the supine position without a pillow and fasting for solids and liquids for half an hour after lumbar puncture; 4-h LSFSL: Lying in the supine position without a pillow and fasting for solids and liquids for four hours after lumbar puncture; HR: Heart rate; LP: Lumbar puncture; LSFSL: Lying in the supine position without a pillow and fasting for solids and liquids; PLPBP: Post-lumbar puncture lower back pain; PLPH: Post-lumbar puncture headache; RR: Respiratory rate; SBP: systolic blood pressure
\end{abstract}

\section{Acknowledgements}

We would like to thank the nurses and doctors working in the Department of Infectious Diseases, Beijing Children's Hospital, who recorded the vital signs and symptom during this study.

Funding

No funding.

\section{Availability of data and materials}

The datasets collected and/or analysed during the current study are available from the corresponding author on reasonable request.

\section{Authors' contributions}

$\mathrm{HB}$ and CTM contributed equally to this work and should be regarded as cofirst authors. LG and PXX are co-corresponding authors and contributed equally to this work. All of the authors had access to the full dataset (including the statistical reports and tables) and take responsibility for the integrity of the data and the accuracy of the data analysis. LG, PXX, HB, CTM, and NXL conceived of and designed the study. HB, CTM, LB, CW and MYQ collected the data and designed the analysis. LG, PXX, HB, CTM and NXL performed the statistical analyses and interpreted the data. CTM, HB and MYQ wrote the first draft of the paper. $L B, C W$, and $N X L$ critically revised the manuscript for important intellectual content. LG, PXX, and CTM reviewed and approved the final report. All authors read and approved the final manuscript.

\section{Ethics approval and consent to participate}

This research was approved (No.2015-2) by the Ethics Committee of Beijing Children's Hospital affiliated with Capital Medical University. The protocol (ID: 20150826) was registered in ClinicalTrials.gov. The study was performed after the children and their patents were fully informed. All participants and/or their parents signed an informed consent form and received written and verbal information before participating in this study. All authors read and approved the final manuscript.

\section{Consent for publication}

Not applicable.

\section{Competing interests}

The authors declare that they have no competing interests.

\section{Publisher's Note}

Springer Nature remains neutral with regard to jurisdictional claims in published maps and institutional affiliations.

Received: 16 March 2018 Accepted: 18 March 2019

Published online: 13 April 2019

References

1. Cooper N. Lumbar puncture. Acute Med. 2001;10(4):188-93. 
2. Stendell L, Foms G, Olsen KS. There is room for improvement in the prevention and treatment of headache after lumbar puncture. Dan Med J. 2012;59(7):A4483.

3. Wang YF, Fu JL, Li JF, et al. Cerebrospinal fluid leakage and headache after lumbar puncture: a prospective non-invasive imaging study. Brain. 2015;138: $1492-8$.

4. Jane A, Mauro V, Gomes B, Marcelo M. Post dural puncture headache: risk factors and clinical features. Cephalalgia. 2012;32(12):916-23.

5. Yan WL, Liu G. Standardization of lumbar puncture in children. Chin J Evid Based Pediatr. 2013;8(3):232-6.

6. Hu YM, Jiang ZF. Zhu Futang practical pediatrics. 8th ed. Beijing: People's Health Publishing House; 2002. p. 164

7. Xiaoli Ge, Jing-Ru Tao,et al. Bayesian estimation on diagnostic performance of Face, Legs, Activity, Cry, and Consolability and Neonatal Infant Pain Scale for infant pain assessment in the absence of a gold standard. Pediatric Anesthesia. 25 (2015):834-39.

8. Pan PH, Fragneto $\mathrm{R}$, Moore $\mathrm{C}$, et al. Incidence of post-dural puncture headache and backache, and success rate of dural puncture: Comparison of two spinal needle designs. South Meal J. 2004;97(4):359-63.

9. Ren B, Li ZP. Clinical analysis of 198 cases with complications after lumbar puncture in children. Chinese journal of practical medicine. 2011;38(1):113.

10. Lavi R, Rowe JM, Avivi I. Lumbar puncture: it is time to change the needle. Eur Neurol. 2010;64(2):108-13.

11. Yan WL, Dou ZZ, Chen TM, et al. Impact of supine recumbency duration on the complications after lumbar puncture in children. J Appl Clin Pediatr. 2014;29(12):914-8

12. Turnbull DK, Shepherd DB. Post-dural puncture headache: pathogenesis, prevention and treatment. 2003:91(5):718-29.

13. Eric D, Jill K, Kayla R, et al. Reducing post lumbar puncture headaches with small traumatic needles. J Clin Neurosci. 2014;21(3):536-7.

14. Yang YX. Achievement of modified lumbar puncture children. Chin J Modern Med. 2007;17(9):1113-4.

15. Shi YQ, Zhou XD. Practical Neurology. 8th ed. Shanghai: Shanghai Science and Technology Publishing House; 2004:64

16. Williams J, Lye DC, Umapath T. Diagnostic lumbar puncture: minimizing complications. Intern Med J. 2008;38(7):587-91

17. Thoennissen J, Herkner H, Lang W, et al. Does bed rest after cervical or lumbar puncture prevent headache? A systematic review and meta-analysis. CMAJ. 2001;165(10):1311-6.

18. Siamak A, Ali D. Evaluation of the effects of resting in appearance of post lumbar puncture headache. Acta Med Iran. 2014;52(1):43-5.

19. Christian $\mathrm{H}$, Jacobs M. Does bed rest prevent post-lumbar puncture headache? Ann Emerg Med. 2012;59(2):139-40.

20. Arevalo-Rodriguez I, Ciapponi A, Roque i, Figuls M, et al. Posture and fluids for preventing post-dural puncture headache. Cochrane Database Syst Rev. (2016, 3):1-30

21. Jin XP, Huang MW, Chen QY, et al. Effect of bed rest time on headache after lumbar puncture. Chinese journal of pain medicine. 2006;(5):314.

Ready to submit your research? Choose BMC and benefit from:

- fast, convenient online submission

- thorough peer review by experienced researchers in your field

- rapid publication on acceptance

- support for research data, including large and complex data types

- gold Open Access which fosters wider collaboration and increased citations

- maximum visibility for your research: over $100 \mathrm{M}$ website views per year

At $\mathrm{BMC}$, research is always in progress.

Learn more biomedcentral.com/submissions 